Volume 9, No.4, July - August 2020

International Journal of Advanced Trends in Computer Science and Engineering

Available Online at http://www.warse.org/IJATCSE/static/pdf/file/ijatcse345942020.pdf

https://doi.org/10.30534/ijatcse/2020/345942020

\title{
Optimal Design the Compliant Bistable Mechanism based on NSGA-II
}

\author{
Ngoc Dang Khoa Tran ${ }^{1}$, Truong Giang Nguyen ${ }^{2}$, Thanh Lam Tran ${ }^{3}$ \\ ${ }^{1}$ Faculty of Mechanical Engineering, Industrial University Of Ho Chi Minh City, Vietnam, \\ tranngocdangkhoa@iuh.edu.vn \\ ${ }^{2}$ Faculty of Mechanical Engineering, Industrial University Of Ho Chi Minh City, Vietnam, \\ nguyentruonggiang@iuh.edu.vn \\ ${ }^{3}$ Faculty of Mechanical Engineering, Ho Chi Minh City of Technology and Education, Vietnam, \\ Corresponding author email: lamtt@hcmute.edu.vn
}

\begin{abstract}
The bistable mechanism has a vital role in precision engineering and robotics with benefits in free friction, no backlash and saves energy. Multi-objective optimization techniques have many applications in design mechanism. NSGA-II is strengthening in quick sorting procedure and elitist methodology. This article introduces an optimization methodology for design compliant bistable mechanisms by employed non dominated sorting genetic algorithm II (NSGA-II). The method predicts the switching forces in forward and backward motion of the bistable mechanism. The program of NSGA-II was executed in Matlab combine with Abaqus to analyze the mechanism. A flowchart of processing is introduced. An example of a bistable mechanism is investigated and results are adopted by applying NSGA-II in order to verify the method.
\end{abstract}

Key words : Bistable mechanism, optimization, NSGA-II, objective function.

\section{INTRODUCTION}

Bistable mechanisms have many applications such as sensors, switches, energy harvester devices and valves, etc. The compliant bistable mechanism has two distinct stable locations formed by store and release energy in motion. It remains in a stable position without any external forces. Many researchers have considered in research the bistable mechanism due to lightweight, no friction, no corrosion and jointless structure [1-3]. Notably, The compressive-stress bucked beams type of bistable mechanism has many studied [4]. Severe geometry designs have been researched. They can employ curve shape [5], slanted straight-line shape [6] or combine many straight lines and segments which use to create compressive and bending loads [7] in the deflection of bistable mechanism.
Many methods to predict and analyze the behavior of compliant bistable mechanisms have been reported. Finite element analysis (FEA) and theoretical modeling are two fundamental methods to adopt these mechanisms' properties. Awtar and Sen [8] present the beam constraint method for investigating a bistable mechanism's characteristics. Hwang et al. [9] introduce an equivalent stiffness model for the chevron bistable mechanism that is suitable for microactuators. Chen et al. [10] develop the compliant bistable mechanism formed by beams that use the Timoshenko beam constraint model. They also developed a chained beam constraint model to study the behavior of the curved bistable mechanism [11].

Many optimized methods are proposed in order to satisfy the eagerly desired to design the compliant bistable mechanisms. Commonly, topology optimization is employed to develop compliant bistable devices. Chen et al. [12] implement the optimal design, which controls the force in the forward and backward movement. Lindgaard and Dahl [13] use compliance and buckling as the objective functions to design the model. The optimal method uses the stress constraint for the compliant device introduced by Silva and Beck [14]. The convergence and stress of these methods are the problems for the optimization algorithms. Chau et al. [15] introduce the LAPO algorithm combined with fuzzy logic and ANFIS in the optimization for predict nonlinear behavior of the bistable mechanism. However, the experiments need to verify the result. Xu et al. [16] used the Euler-Bernoulli beam bending theory to approve the elastic characteristic and particle swarm optimization method for the nonlinear model. Taguchi algorithm for optimization are also engaged to design compliant mechanism [17, 18], nonlinear behavior should be investigated. Genetic algorithm (GA) has much utilization in engineering [19, 20], especially non dominated sorting genetic algorithm II (NSGA-II) is widespread in design mechanism [21-23]. The method developed from the Genetic algorithm with multi-objective function, which advances for fast nondominated sorting, crowding distance method and elitism methodology increased the convergence and robustness. 
This study introduces the process of NSGA-II applies for design bistable mechanism with nonlinear behavior. The switching forces are the objective functions to predict then bistable properties. The combination of Matlab and Abaqus is used for optimal design.

\section{DESIGN}

A schematic of the compliant bistable mechanism is illustrated in figure 1 . The model includes four beams with one fixed end of each beam and the other end, they connect with the center mas. In each beam, it consists of two thin beams and one reinforcement beam. Figure 2 shows the details of one beam, the design parameters of the beam are also performed in this figure. Five segments construct it, segments 1, 2, 4 and 5 are the thin beams and have the same width, $w_{1}$. Segment 3 has the width $w_{3}$. Segment 5 is the reinforcement beam. The length of each beam is also performed in this figure. $\theta_{1}, \theta_{2}$ and $\theta_{5}$ are the inclination angle of the segment and the horizontal axis. The inclination of segments 3 and 4 is the same as segment 2 . The model has a uniform in thickness.

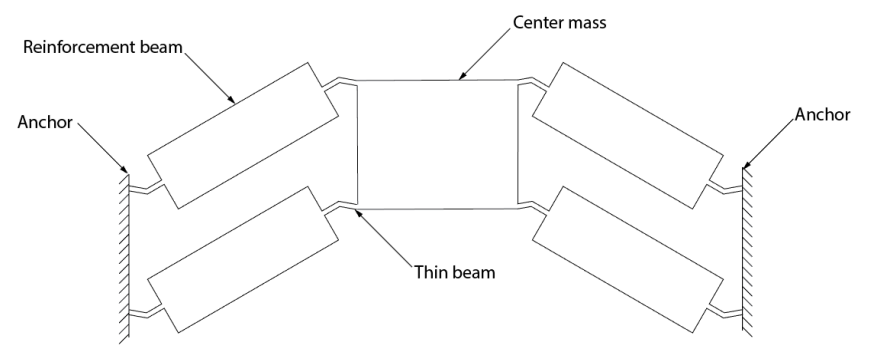

Figure 1: Model of compliant bistable mechanism.

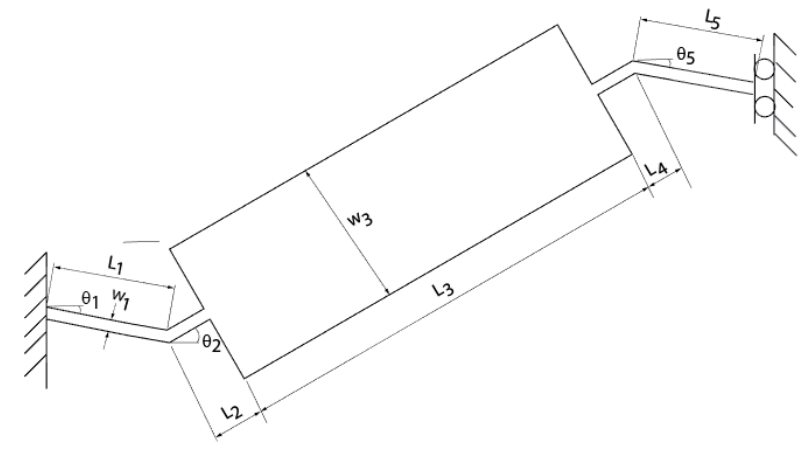

Figure 2: Design parameters of each beam.

The compliant bistable mechanism has nonlinear behavior featured by force and displacement relation. Operation of the bistable mechanism is shown in figure 3. Figure 3a demonstrates that the model stays in the first stable position. When When applying a displacement into the center mass, center mass moves follow the vertical axis, center mass moves follow the vertical axis. Figure $3 \mathrm{~b}$ shows the second stable position of the model. A characteristic of the property is shown in figure 4, a nonlinear of the stiffness of the model is demonstrated. Initial condition, the center mass stays in the first stable position $S_{l}$, when the displacement increases, the reaction force in the fixed end of beams also develops and reach maximum force in $F_{\max }$, the displacement continually increases, the force goes down and turns into a negative force and archives the minimum force at $F_{\min }$. After that, the displacement increases until it reaches the second stable position $S_{2}$. At this position, the model becomes the stable stage and it consumes zero power to keep this stage.

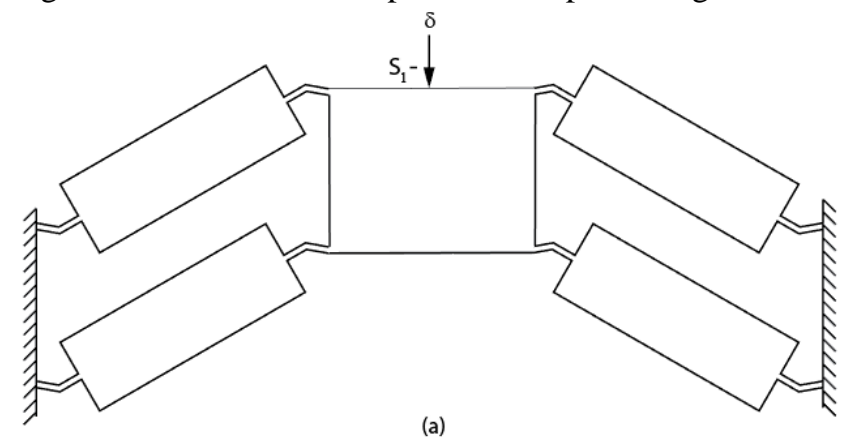

(a)

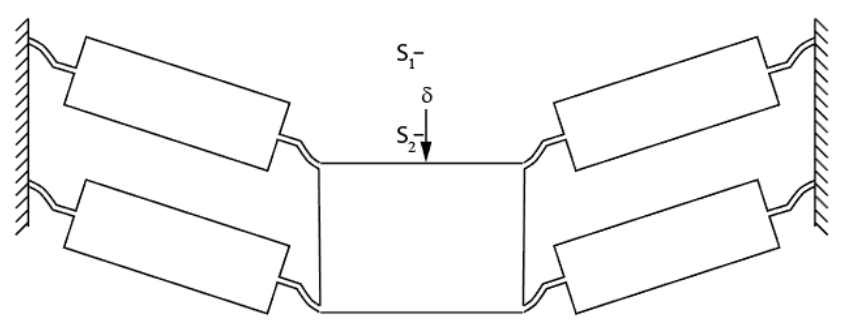

(b)

Figure 3: Operation of bistable mechanism.

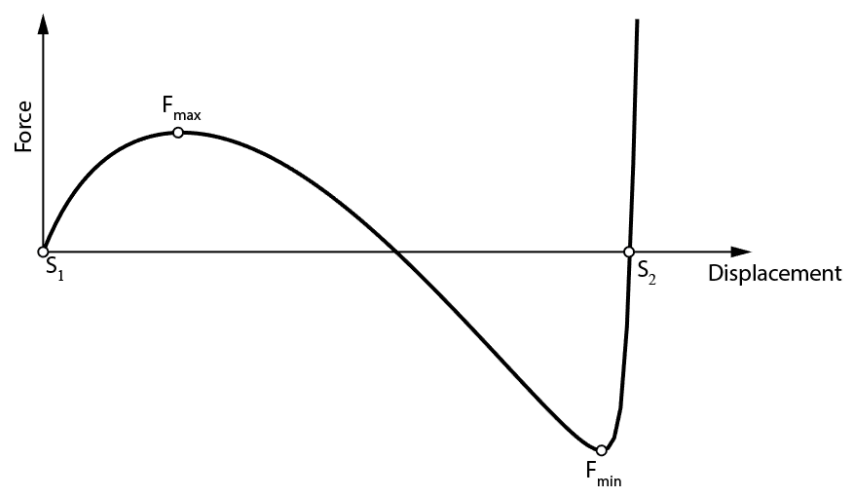

Figure 4: A foce - displacement curve of bistable mechanism

\section{OPTIMAL METHODOLOGY}

The NSGA-II method is applied to find the best design with the objective function is $F_{\max }, F_{\min }$ and $S_{2}$. The process of the method is performed in figure 5. In the beginning, the NSGA-II code is composed of the Matlab program, the declaration about the fixed and variable design parameters, the objective functions and other conditions are put in this code. When Matlab runs, the geometry model is created and writes the Abaqus file. Matlab calls the Abaqus program to mesh and analyze the model. The force-displacement curve is 
created. Then, Abaqus return the results to Matlab so that it can find the maximum force $F_{\max }$, minimum force $F_{\min }$ and second stable position $S_{2}$. Based on three parameters, the NSGA-II process executes and evaluates the results.

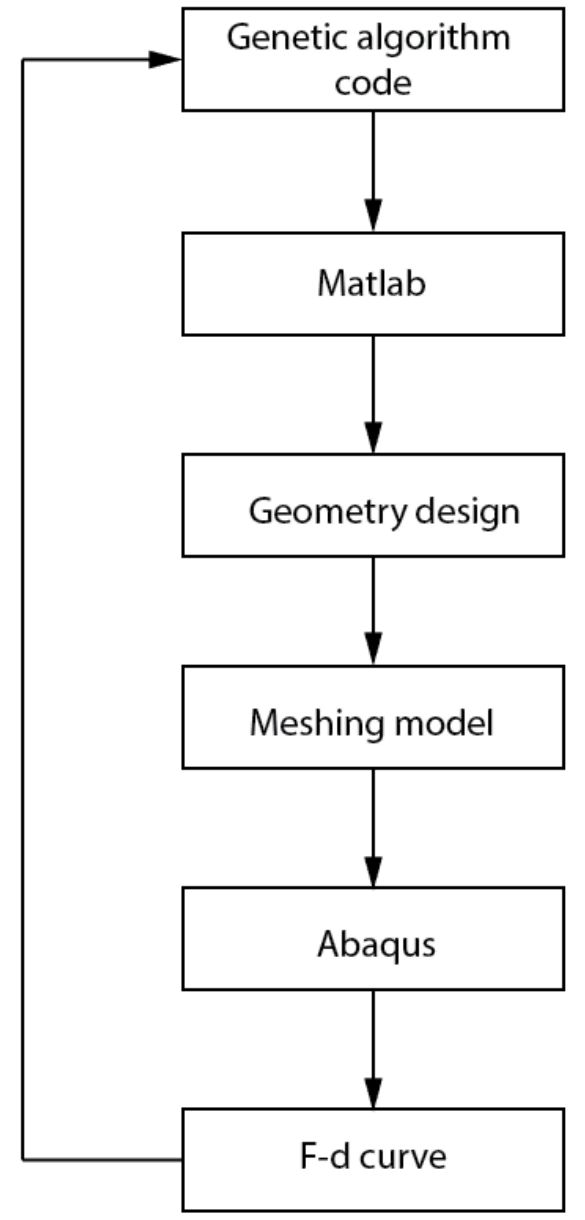

Figure 5: Process of the optimal method

NSGA-II is separated into two parts. Part one creates the individual population, the flowchart is shown in figure 6 . In this part, a generation with size $\mathrm{N}$ is created, random design parameters are chosen to a role as an individual ith generation. Based on this parameter, the Abaqus program executes and gives three parameters $F_{\max }, F_{\min }$ and $S_{2}$ to Matlab. The NSGA-II check nonlinear behavior is satisfied. If the program refuses the results, new design parameters are created and analyze again. When the condition is satisfied, the calculation of objective functions is implemented. The comparison of objective function enhances the design. When the program finds enough generation, part two is executed, it is the genetic algorithm. Figure 7 shows the flowchart of this process, jth is the current generation and $\mathrm{M}$ is the size of the generation. The genetic operator for each generation creates offsprings. Each offspring evaluate the nonlinear property and objective functions. Then combine generation has occurred and nondominated sorting and create the $(j+1)$ th generation. When the generation is enough, the program stops and gives the final design with the parameters desired the best objective function.

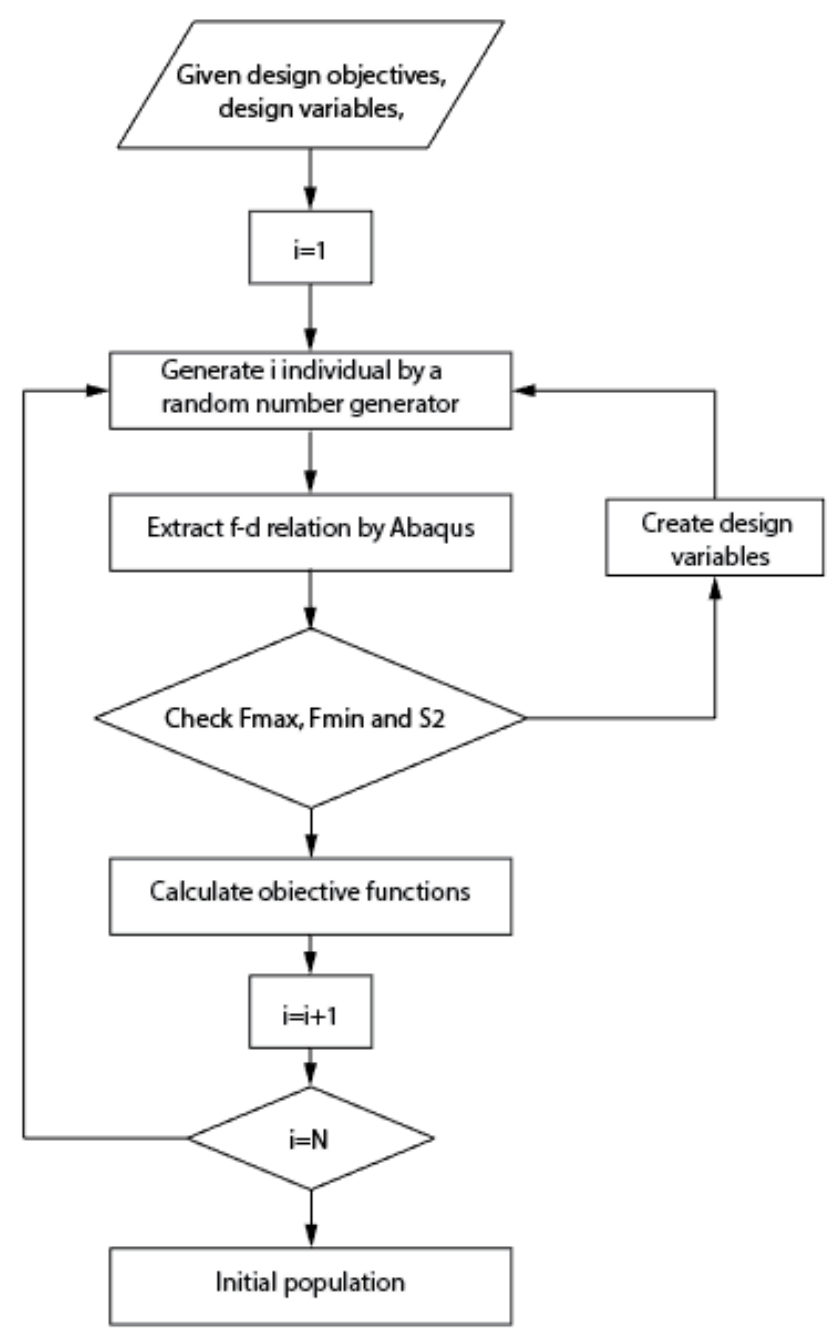

Figure 6: Flowchart of loop for creation of initial popular.

\section{RESULTS AND DICUSSION}

An example of a design compliant bistable mechanism is investigated with the variable parameters are performed in Table 1. The length of segment 4 is equal to the length of segment $2\left(L_{2}=L_{4}\right)$ and The length of segment 5 is equal to the length of segment $1\left(L_{1}=L_{5}\right)$. The requirement of the design is maximum force, minimum force and second stable position are $3700(\mathrm{mN}),-3700(\mathrm{mN})$ and $8.2(\mathrm{~mm})$, respectively. The lower and upper bound are also shows in this table. The units of each parameter follow SI units and are also shown in the table. The thickness of the model is $5 \mathrm{~mm}$ The thermoplastic of polyoxymethylene (POM) is taken as a material for the design. The Young's modulus is given $2.2 \mathrm{Gpa}$ and the Poisson ratio is 0.68 . The model is a uniform thickness parameter, is $5 \mathrm{~mm}$. 


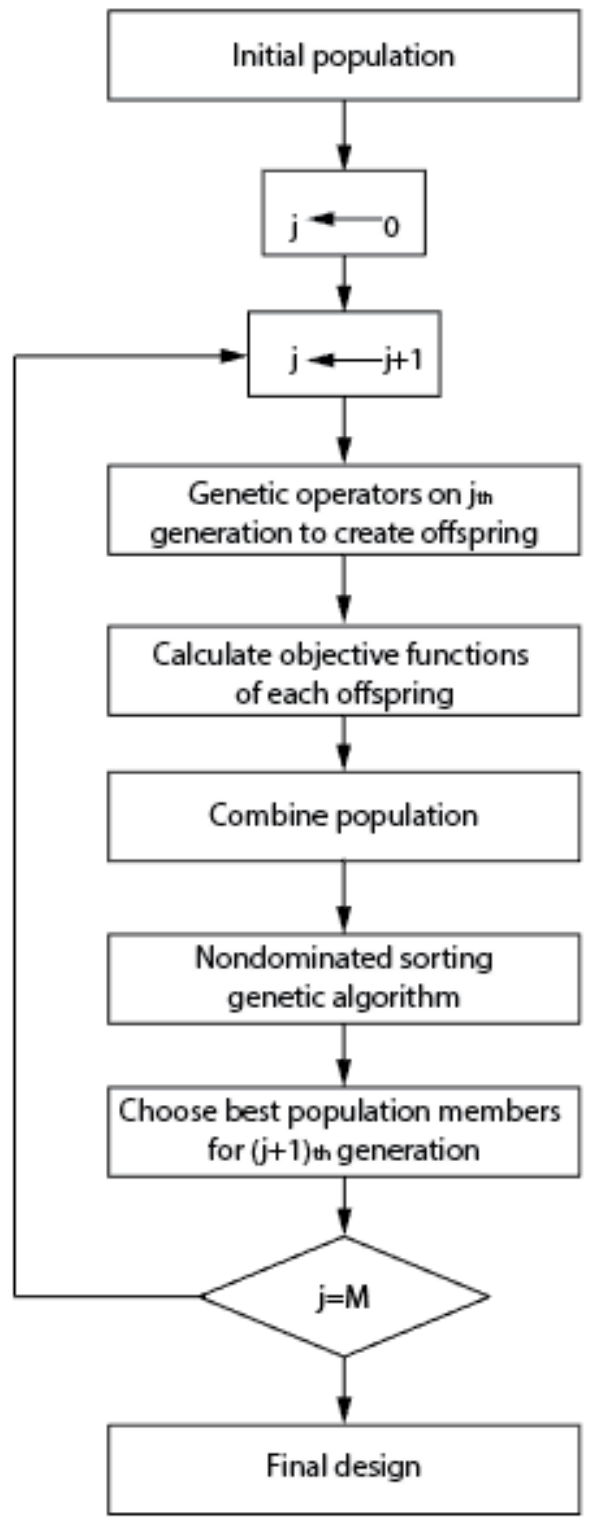

Figure 7: Flowchart of genetic algorithm.

Table 1: Variable parameters for the bistable model.

\begin{tabular}{|c|c|c|}
\hline Variables & Lower bound & Upper bound \\
\hline$L_{1}(\mathrm{~mm})$ & 6 & 15 \\
\hline$L_{2}(\mathrm{~mm})$ & 4 & 10 \\
\hline$L_{3}(\mathrm{~mm})$ & 20 & 70 \\
\hline$\theta_{1}($ degree $)$ & -5 & 5 \\
\hline$\theta_{2}$ (degree) & 0 & 10 \\
\hline$\theta_{5}$ (degree) & -5 & 5 \\
\hline
\end{tabular}

The objective functions of the mechanism are:

Min $\mid$ Fmax-3700|

Min $\mid$ Fmin-3700|

$\operatorname{Min}|S 2-8.2|$

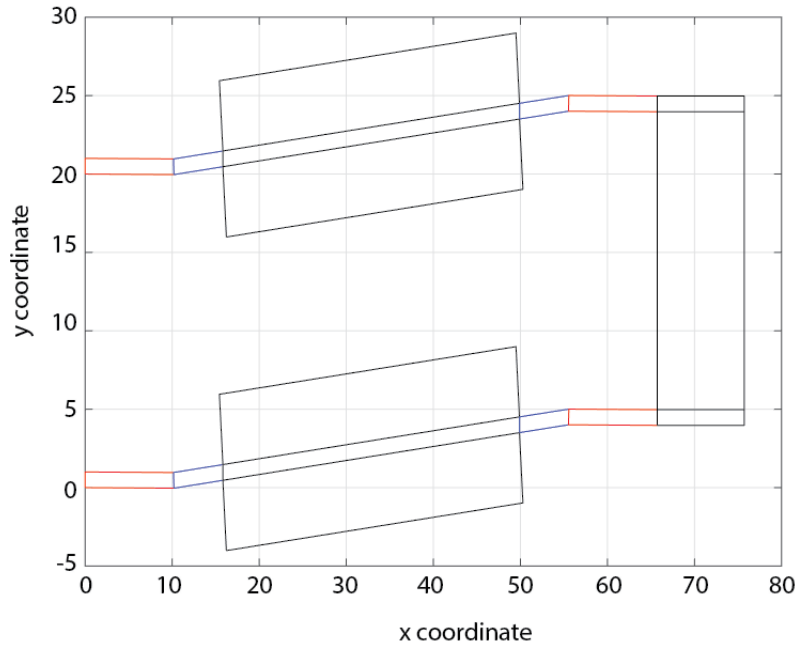

Figure 8: Geometry of half model.

All parameters are designed is put in Matlab code. When running the program, random parameters is created form table 1 . The geometry of the bistable mechanism is formed. Due to the symmetry condition, a half model is applied to analyze. Figure 8 shows the geometry of the half model.

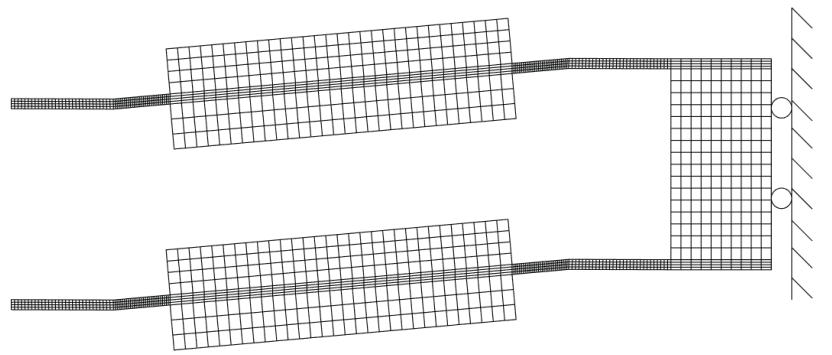

Figure 9: A mesh of half model.

Based on the geometry model, a mesh file is created and called the Abaqus program to execute. A mesh of half model is shown in figure 9. The center mass has the boundary condition is the roller, allows center mass to move in the horizontal axis and limit the vertical axis. The other end of the beams is fixed. After the Abaqus program analyzes the model. The result of the model is performed in figure 10 . Maximum force, minimum force and second stable position are calculated and put in Matlab file and NSGA-II process to generate the individual and generation. A 15 individual and 40th generation are set up in this example. 


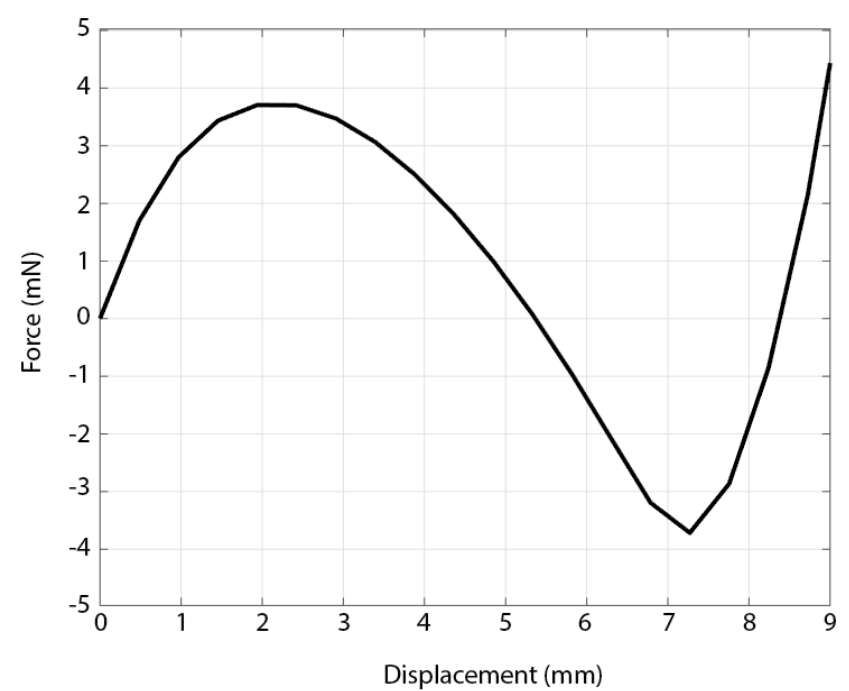

Figure 10: Force - displacement of the model.

After NSGA-II finishes the program, the result of each generation is created. Figure 11 performs the individual of generation 20th, 30th and 40th. Each generation has 15 individuals is generated. At generation 40th, an optimal solution is selected that it has the smallest objective function. The design parameters of this model are demonstrated in table 2. The maximum force is $3706(\mathrm{mN})$ and the minimum force is $-3716(\mathrm{mN})$, the second stable position is 8.23 . The result is closed to the requirement of the model. The error is smaller than $2 \%$.

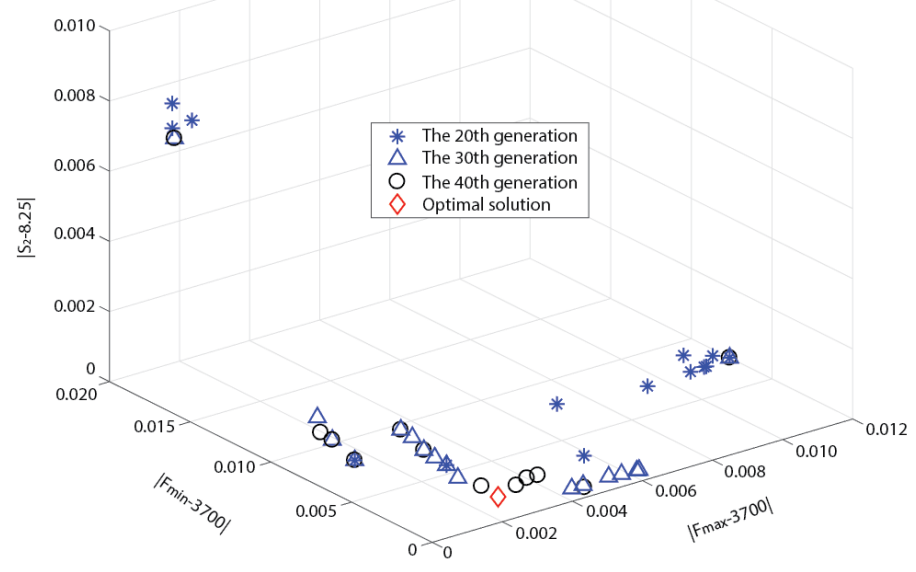

Figure 11: Generation $20^{\text {th }}, 30^{\text {th }}, 40^{\text {th }}$, of the NSGA-II.

\section{CONCLUSION}

An application of NSGA-II in design a compliant bistable mechanism is described. The bistable mechanism with nonlinear behavior is introduced and it constructed by four beams with five segments of each beam. The combination of the Matlab and Abaqus program is engaged in the NSGA-II process. An example of a design bistable mechanism with three objective functions is examined. NSGA-II obtains the results that have small errors. It is an adequate method to design the bistable mechanism.

Table 2: Results of optimal solution.

\begin{tabular}{|c|c|}
\hline Variables & Values \\
\hline$L_{1}(\mathrm{~mm})$ & 10.2 \\
\hline$L_{2}(\mathrm{~mm})$ & 5.65 \\
\hline$L_{3}(\mathrm{~mm})$ & 34.2 \\
\hline$\theta_{1}($ degree$)$ & -0.2 \\
\hline$\theta_{2}($ degree $)$ & 5.1 \\
\hline$\theta_{5}($ degree $)$ & -0.1 \\
\hline$F_{\max }(\mathrm{mN})$ & 3706 \\
\hline$F_{\min }(\mathrm{mN})$ & -3716 \\
\hline$S_{2}(\mathrm{~mm})$ & 8.23 \\
\hline
\end{tabular}

\section{REFERENCES}

1. D. L. Wilcox and L. L. Howell. Fully compliant tensural bistable micromechanisms (FTBM), Journal Of Microelectromechanical Systems, vol. 14, no. 6, December 2005.

2. J. Zhao, Y. Yang, K. Fan, P. Hu, H. Wang, A bistable threshold accelerometer with fully compliant clampedclamped mechanism, IEEE Sensors Journal, issue 5, 2010.

3. M.Vangboy and Y. Backlund. A lateral symmetrically bistable buckled beam, J. Micromech. Microeng., Vol. 8 pp. 29-32, 1998. https://doi.org/10.1088/0960-1317/8/1/005

4. P. Cazottes, A. Fernandes, J. Pouget and M. Hafez. Bistable buckled beam: modeling of actuating force and experimental validations, Journal of Mechanical Design, vol. 131, 2009.

5. J. Qiu and J. H. Lang. A curved-beam bistable mechanism, Journal of Microelectromechanical Systems, vol. 13, no. 2, April 2004

6. B. D. Jensen, M. B. Parkinson, K. Kurabayashi, L. L. Howell and M. S. Baker. Design optimization of a fully-compliant bistable micro-mechanism, $A S M E$ International Mechanical Engineering Congress and Exposition, Newyork, November 2001.

7. T. H. Ngo, H. V. Tran, T. A. Nguyen, T. P. Dao and D. A. Wang. Design and kinetostatic modeling of a compliant gripper for grasp and autonomous release of objects, Advanced Robotics, issue 14, vol. 32,pp. 717-735, 2018.

8. S. Awtar and S. Sen. A generalized constraint model for two-dimensional beam flexures: Nonlinear loaddisplacement formulation, J. Mech. Des. Trans. ASME, vol. 132, 2010. https://doi.org/10.1115/1.4002006 
9. I. H. Hwang, Y. S. Shim and J. H. Lee. Modeling and experimental characterization of the chevron-type bi-stable microactuator, Journal Of Micromechanics And Microengineering, vol.13, pp. 948-954, 2003

10. G. Chen and F. Ma. Kinetostatic modeling of fully compliant bistable mechanisms using Timoshenko beam constraint model, Journal of Mechanical Design, vol. 137, 2015.

11. G. Chen, F. Ma, G. Hao and W. Zhu. Modeling large deflections of initially curved beams in compliant mechanisms using chained beam constraint model, $J$. Mech. Rob., vol.11, 2019.

12. Q. Chen, X. Zhang, H. Zhang, B. Zhu and B. Chen. Topology optimization of bistable mechanisms with maximized differences between switching forces in forward and backward direction, Mechanism and Machine Theory, vol. 139, pp. 131-143, 2019.

13. E. Lindgaard and J. Dahl. On compliance and buckling objective functions in topology optimization of snap-through problems, Struct. Multidiscip. Optim., vol. 47, pp. 409-421, 2013. https://doi.org/10.1007/s00158-012-0832-2

14. G. A. Silva, A. T. Beck and O. Sigmund. Stress-constrained topology optimization considering uniform manufacturing uncertainties, Comput. Methods Appl. Mech. Eng., vol. 344, pp. 512-537, 2019

15. N. L. Chau, N. T. Tran and T. P. Dao. A multi-response optimal design of bistable compliant mechanism using efficient approach of desirability, fuzzy logic, ANFIS and LAPO algorithm, Applied Soft Computing, vol. 94, 2020

16. Q. $\mathrm{Xu}$ and Y. Li. Analytical modeling, optimization and testing of a compound bridge-type compliant displacement amplifier, Mechanism and Machine Theory, vol 46, pp.183-200, 2011.

17. T. P. Dao, S. C. Huang and P.T. Thang. Hybrid Taguchi-cuckoo search algorithm for optimization of a compliant focus positioning platform, Applied Soft Computing, vol. 57, 2017.

18. G. Chandrasekaran, R.Saravanan and J.Maniraj. Multiobjective Optimisation of Bevel Gear Pair design using NSGA-II, Materialstoday proceeding, V. 16, Part 2, pp. 351-360, 2019.

19. A. A. M Zahir, S. S. N Alhady, W. A. F. W Othman, Z. Low and A. A. A Wahab. Genetic Algorithm Optimization and Implementation of Velocity Control PI Controller for Cart Follower Application, International Journal of Advanced Trends in Computer Science and Engineering, vol. 8, no. 5, 2019. https://doi.org/10.30534/ijatcse/2019/12852019

20. A. J. A. Abello, G. F. Y. Domingo, M. J. T. Joven, S. A. S. Malubay. Power measurement model optimization using Matlab, International Journal of Advanced Trends in Computer Science and Engineering, vol. 8, no. 3, 2019. https://doi.org/10.30534/ijatcse/2019/31832019
21. M. Mahmoudi, A. Fatehi, H. Jafari and E. Karimi. Multi-objective micro-grid design by NSGA-II considering both islanded and grid-connected modes, IEEE Texas Power and Energy Conference (TPEC), USA, 2018.

22. T. P. Dao, S. C. Huang and N. L. Chau. Robust parameter design for a compliant microgripper based on hybrid Taguchi-differential evolution algorithm, Microsyst. Technol, 2017.

23. T. P. Dao, S. C. Huang and P.T. Thang. Hybrid Taguchi-cuckoo search algorithm for optimization of a compliant focus positioning platform, Applied Soft Computing, vol. 57, 2017. 